\title{
In-hospital prognosis of first-ever noncardiogenic ischemic stroke in patients with and without indication for prestroke antiplatelet therapy: Chinese Stroke Center Alliance
}

\author{
Yu-Yuan $\mathrm{Xu}^{1}{ }^{\wedge}$, Hong-Qiu Gu ${ }^{1}$, Zi-Xiao Li ${ }^{2}$, Yun-Yun Xiong ${ }^{1}$, Qi Zhou ${ }^{1}$, Li-Ping Liu ${ }^{2}$, Xing-Quan Zhao ${ }^{2}$, \\ Yi-Long Wang ${ }^{2,3}$, Xia Meng ${ }^{1}$, Yong-Jun Wang ${ }^{2,3}$; on behalf of the CSCA Investigators \\ ${ }^{1}$ China National Clinical Research Center for Neurological Diseases, Beijing Tiantan Hospital, Capital Medical University, Beijing, China; \\ ${ }^{2}$ Department of Neurology, Beijing Tiantan Hospital, Capital Medical University, Beijing, China; ${ }^{3}$ Beijing Key Laboratory of Translational Medicine \\ for Cerebrovascular Disease, Beijing, China \\ Contributions: (I) Conception and design: YY Xu, YY Xiong, ZX Li; (II) Administrative support: X Meng, ZX Li; (III) Provision of study materials or \\ patients: X Meng, ZX Li; (IV) Collection and assembly of data: X Meng, XQ Zhao, LP Liu, YL Wang; (V) Data analysis and interpretation: HQ Gu, \\ Q Zhou; (VI) Manuscript writing: All authors; (VII) Final approval of manuscript: All authors. \\ Correspondence to: Dr. Yong-Jun Wang. No. 119 South 4th Ring West Road, Fengtai District, Beijing 100070, China. \\ Email: yongjunwang@ncrend.org.cn.
}

Background: It is unknown about the influence of prestroke antiplatelet use on early outcomes in patients with and without the indication. We aimed to evaluate the in-hospital prognosis of first-ever noncardiogenic ischemic stroke patients with and without indications of antiplatelet use for primary prevention.

Methods: This was a retrospective, observational study based on a prospective hospital-based registry (Chinese Stroke Center Alliance). Using the data with 436,660 first-ever noncardiogenic acute ischemic strokes recorded from Aug 1, 2015, to July 31, 2019, from 1,453 hospitals in China, we examined the associations between the indication for prestroke antiplatelet use and in-hospital clinical outcomes.

Results: Among 436,660 first-ever noncardiogenic ischemic stroke patients, 42,409 patients (9.7\%) had a documented previous vascular indication and 394,251 (90.3\%) did not. Compared to those without, patients with the indication were associated with increased prevalence of in-hospital morbid conditions, including stroke severity (OR 2.71; 95\% CI: 2.62-2.81; P<0.0001), length of stay >14 days (OR 1.16; 95\% CI: $1.13-$ 1.19; $\mathrm{P}<0.0001$ ), mortality (OR 2.20; 95\% CI: 1.96-2.46, $\mathrm{P}<0.0001$ ), and recurrence of ischemic stroke and transient ischemic attack (TIA) (OR 1.5; 95\% CI: 1.43-1.59, $\mathrm{P}<0.0001)$. Among patients without indication, prestroke antiplatelet use was associated with lower mortality (OR 0.73, 95\% CI: 0.56-0.96; P=0.0221); while among patients with indication, those receiving prestroke antiplatelet had lower odds ratios in stroke severity $(\mathrm{P}<0.0001)$ and disability $(\mathrm{P}=0.0003)$ than those who not.

Conclusions: Patients with indications of prestroke antiplatelet use were more likely to have unfavorable outcomes than those without. Prestroke antiplatelet might be associated with lower mortality, less disability, and less stroke severity in certain population groups. Future studies to improve risk prediction rules are needed to guide effective primary prevention for ischemic stroke.

Keywords: Antiplatelet therapy; ischemic stroke; prognosis; primary prevention

Submitted Sep 08, 2020. Accepted for publication Dec 18, 2020.

doi: 10.21037/atm-20-7902

View this article at: http://dx.doi.org/10.21037/atm-20-7902

^ ORCID: 0000-0002-7109-1283. 


\section{Introduction}

Stroke is the first leading cause of mortality and disability in China. The stroke burden in China has increased over the past 30 years and remains particularly high in rural areas (1).

Prevention is vital to reduce the disease burden and antiplatelet agents are one of the mainstay therapies for primary and secondary stroke prevention. Although antiplatelet agents play an important role in primary prevention, it remains controversial whether the previous therapy can improve outcomes of acute ischemic stroke. Several previous studies pointed out the fact that prestroke antiplatelet use, especially aspirin, could benefit the vital prognosis of stroke patients $(2,3)$. However, a 2016 metaanalysis of 11 trials that included individual patient level data among 118,445 men and women who were randomly assigned to either aspirin (at doses between 50 and $500 \mathrm{mg}$ daily) or placebo found no significant benefit on nonfatal stroke (relative risk 0.95, 95\% CI: 0.85-1.06) (4). According to the guideline, for people with risk factors, the application of aspirin as the primary prevention of cardiovascular disease (CVD) requires careful consideration $(5,6)$.

Therefore, our study aims to (I) describe characteristics of first-ever noncardiogenic ischemic stroke patients by whether an indication of antiplatelet for primary prevention was present or not; (II) examine the difference of inhospital prognosis of patients with and without indication of previous antiplatelet use; (III) study the in-hospital prognostic value of previous antiplatelet use on stroke severity, mortality, disability, hospital length of stay and inhospital recurrence of ischemic stroke or transient ischemic attack (TIA).

We present the following article in accordance with the STROBE reporting checklist (available at http://dx.doi. org/10.21037/atm-20-7902).

\section{Methods}

\section{Cobort}

We derived data from the Chinese Stroke Center Alliance (CSCA) database. The protocol for case identification and data collection have been previously reported elsewhere (7). Briefly, the CSCA is a national, hospitalbased, voluntary, and multifaceted intervention, and also a continuous quality improvement initiative. It provides a unique platform to develop stroke centers and to improve stroke care quality and clinical outcomes. This program is available to all Chinese secondary and tertiary grade hospitals. Hospitals continued to join the program in a staggered manner. Patients were enrolled if they had a primary diagnosis of stroke or TIA and were then confirmed by brain computerized tomography (CT) or magnetic resonance imaging (MRI). Data were collected by trained hospital personnel using a web-based Patient Management Tool (Medicine Innovation Research Center, Beijing, China). The China National Clinical Research Center for Neurological Diseases (NCRCND) serves as the data analysis center and has an agreement to analyze the aggregate deidentified data for care quality feedback and research purposes.

The study was conducted in accordance with the Declaration of Helsinki (as revised in 2013). The study was approved by the central Institutional Ethics Board at Beijing Tiantan Hospital (No.: KY 2018-061-02) and individual consent for this retrospective analysis was waived.

\section{Study population}

The population of this study consists of all stroke/TIA patients enrolled from secondary or tertiary hospitals across 31 provinces, autonomous regions, or municipalities in Mainland China participating in the CSCA program from Aug 1, 2015, to July 31, 2019. A total of 838,229 patients were recruited consecutively from all 1,476 hospitals in CSCA if they met the following criteria: (I) age $>18$ years; (II) diagnosis within 7 days of the index event of ischemic stroke; (III) informed consent provided by the patient or a legally authorized representative. Patients with cardiogenic stroke or with a history of stroke or TIA, patients receiving prestroke anticoagulants, and patients missing information on the National Institute of Health Stroke Scale (NIHSS) score were excluded. After these exclusions, our primary study population consisted of 436,660 patients from 1,453 hospitals in China (Figure 1).

\section{Data collection and management}

Patient information including demographics, risk factors, comorbidities, medications, discharge status, and hospitallevel characteristics was systematically collected during hospitalization and at discharge by trained research coordinators at each participating hospital.

Patients were considered to indicate for prestroke antiplatelet therapy if their medical history included coronary artery disease, myocardial infarction, or atrial fibrillation (AF) (8). Patients were categorized as with and without 


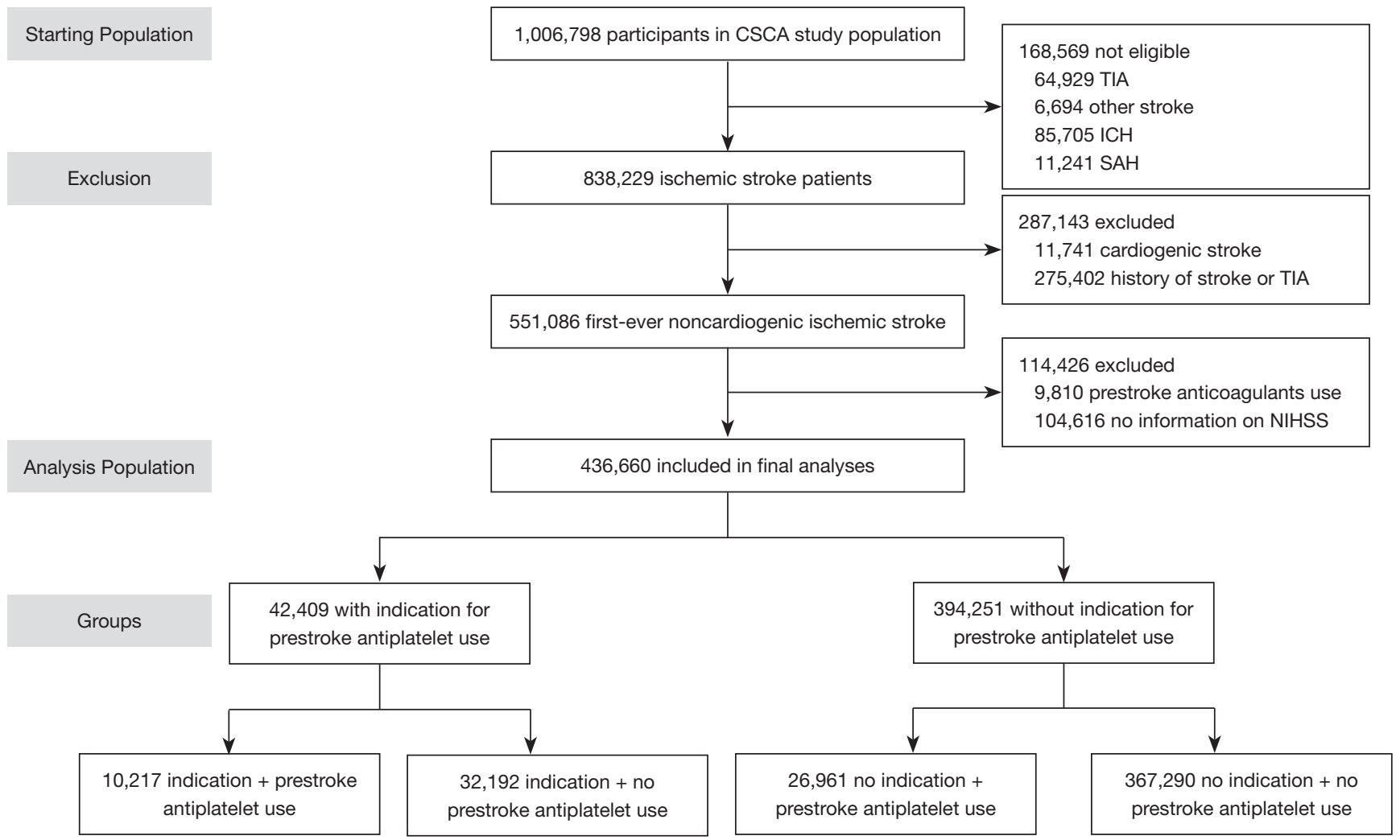

Figure 1 Study population. Indication for prestrike antiplatelet use: with medical history included coronary artery disease, myocardial infarction, or atrial fibrillation. CSCA, the Chinese Stroke Center Alliance; TIA, transient ischemic attack; ICH, intracranial hemorrhage; SAH, subarachnoid hemorrhage; NIHSS, National Institute of Health Stroke Scale.

indication groups. Prestroke antiplatelet use was defined as using antiplatelet agents (including aspirin, clopidogrel, ticagrelor, etc.) continuously for 2 weeks in recent 6 months. Based on the indication and prestroke antiplatelet use, patients were further divided into 4 subgroups: indication + prestroke antiplatelet use, indication + no prestroke antiplatelet use, no indication + prestroke antiplatelet use, no indication + no prestroke antiplatelet use.

\section{In-hospital outcomes}

The National Institute of Health Stroke Scale (NIHSS) score on administration was used as an index of stroke severity and the modified Rankin Scale (mRS) score at discharge as an index of functional outcome. The initial stroke severity was categorized as low (NIHSS $\leq 15)$ and high (NIHSS 16-42) levels. Disability at the time of discharge was defined as $m R S$ score $\geq 2$. Our study examined patient-relevant outcomes of NIHSS on administration,
$\mathrm{mRS}$ at discharge, hospital length of stay for over 14 days, in-hospital mortality, in-hospital intracranial hemorrhage, and in-hospital recurrence of ischemic stroke and TIA.

\section{Statistical analysis}

Categorical variables were reported as absolute numbers with percentages. Means (standard deviations) and medians $\left(25^{\text {th }}-75^{\text {th }}\right.$ percentiles) were used to describe the distribution of continuous variables. Multivariable logistic regression analyses investigated the associations between indications for prestroke antiplatelet therapy and each outcome, adjusted for age, gender, medical history, baseline NIHSS, smoking, and poststroke medications. Multivariable logistic regression was used to analyze the difference of outcomes among the four subgroups, with the "no indication + no prestroke antiplatelet use" subgroup selected as the reference. Age, gender, carotid stenosis, peripheral vascular disease, hypertension, dyslipidemia, and diabetes mellitus 
were introduced in the model.

All $\mathrm{P}$ values are 2 sided, with $\mathrm{P}<0.05$ considered statistically significant. All statistical analyses were performed using SAS Version 9.4 software (SAS Institute, Cary, NC, USA). A SAS macro named \%ggBaseline was used to analyze and report baseline characteristics automatically (9).

\section{Results}

\section{Characteristics at the recruitment of study participants}

Among 436,660 first-ever noncardiogenic ischemic stroke patients (Figure 1), 42,409 (9.7\%) indicated prestroke antiplatelet therapy. $8.5 \%$ of patients were not on any antiplatelet therapy before their incident stroke, with $24.1 \%$ and $6.8 \%$, respectively, among patients with and without indications. Table 1 displayed the demographic, clinical, and hospital characteristics according to groups with and without indication for prestroke antiplatelet use. Patients with an indication for prestroke antiplatelet use were more likely to be female (46.2\% vs. 36.6\%) and older $(71.3 \pm 11.0$ vs. $64.8 \pm 12.3)$, and had a greater prevalence of cardiovascular risk factors (carotid stenosis, peripheral vascular disease, hypertension, dyslipidemia, and diabetes mellitus), and were associated with previous medication (antiplatelets, anti-hypertensives, lipid-lowering medication, and antidiabetics) before the index stroke. After the firstever stroke, the proportions of patients on treatment among two groups (81.6\% vs. 86.6\%) reflected antiplatelet use for secondary prevention. ASD of all variables above crossed the border of $10 \%$.

\section{Indications for prestroke antiplatelet use and post-stroke outcomes}

The number and rates of in-hospital mortality, stroke recurrence, and other outcomes were listed in Table 2 according to groups with and without indication. Overall rates of in-hospital severe stroke (NIHSS 16-42), hospital stay $>14 \mathrm{~d}$, stroke or TIA recurrence, hemorrhagic stroke and mortality were $4.3 \%(\mathrm{~N}=18,919), 19.5 \%(\mathrm{~N}=85,126)$, $2.5 \%(\mathrm{~N}=11,028), 0.8 \%(\mathrm{~N}=3,516)$, and $0.4 \%(\mathrm{~N}=1,597)$, respectively. After adjusting for potential confounders, we found that patients with an indication were associated with increased prevalence of many morbid conditions including initial stroke severity (NIHSS 16-42) (OR 2.71; 95\% CI: $2.62-2.81 ; \mathrm{P}<0.0001)$, the length of stay $>14 \mathrm{~d}(\mathrm{OR} 1.16$;
95\% CI: 1.13-1.19; $\mathrm{P}<0.0001$ ), mortality (OR 2.2; $95 \%$ CI: 1.96-2.46, $\mathrm{P}<0.0001)$, and in-hospital recurrence of ischemic stroke and TIA (OR 1.5; 95\% CI: 1.43-1.59, $\mathrm{P}<0.0001)$.

\section{Prestroke antiplatelet use and poststroke outcomes}

Table 3 showed the regression model results respectively, by prestroke antiplatelet use and indication. Among patients without indication, prestroke antiplatelet use was associated with lower mortality (OR 0.73, 95\% CI: 0.56-0.96; $\mathrm{P}=0.0221$ ), but longer hospital stay (OR 1.03, 95\% CI: 1-1.07; $\mathrm{P}=0.0327)$, and more recurrence of ischemic stroke and TIA (OR 1.59, 95\% CI: 1.48-1.70; $\mathrm{P}<0.0001$ ); while among patients with indication, there was a marginally significant inverse association of prestroke antiplatelet use with NIHSS $\geq 16(\mathrm{P}<0.0001)$ and $\mathrm{mRS} \geq 2(\mathrm{P}=0.0003)$, and prestroke antiplatelet use was not associated with length of hospital stay, recurrence of ischemic stroke and TIA, or mortality.

\section{Discussion}

This national hospital-based study including over 1 million stroke patients across China indicated that, in China, more than $90 \%$ of the stroke patients occurred among those without any indication for antiplatelet use based on the current primary prevention guideline; among those who met the indication, only a quarter of the patients were on treatment. Furthermore, in this study, patients with indications were more likely to have unfavorable outcomes than those without, probably due to the high baseline risk for CVD. Prestroke antiplatelet use was, however, only associated with NIHSS and mRS score in the highrisk group. With the large sample size of well-phenotyped stroke patients included, and comprehensive prognostic characteristics recorded, our study may have important clinical implications.

First, in China, $90.3 \%$ of patients were classified as non-indication but finally developed ischemic stroke. The paradox of high risk of stroke with low risk of CVD could be observed in Asian and other populations. Previous studies inferred that it was not due to atherosclerosis in the major cerebral arteries but lesions in the small intracerebral arteries (10). Risk factors that enhanced thrombosis and reduced fibrinolysis were also capable of explaining the paradoxical occurrence of the incidence of coronary heart disease and stroke in certain populations (11). 
Table 1 Baseline characteristics

\begin{tabular}{|c|c|c|c|c|}
\hline Variables & $\begin{array}{c}\text { Total } \\
(\mathrm{N}=436,660)\end{array}$ & $\begin{array}{c}\text { Indication for antiplatelet } \\
\text { use, } N=42,409(9.7 \%)\end{array}$ & $\begin{array}{c}\text { No indication for antiplatelet } \\
\text { use, } N=394,251(90.3 \%)\end{array}$ & $\begin{array}{l}\text { ASD/HL } \\
\text { estimator }\end{array}$ \\
\hline Age, yrs & $65.4 \pm 12.4$ & $71.3 \pm 11.0$ & $64.8 \pm 12.3$ & 55.7 \\
\hline Sex, female, No. (\%) & $163,822(37.5)$ & $19,614(46.2)$ & $144,208(36.6)$ & 19.6 \\
\hline $\mathrm{BMI}, \mathrm{kg} / \mathrm{m}^{2}$ & $23.9 \pm 4.0$ & $23.9 \pm 4.0$ & $23.9 \pm 4.0$ & 0.0 \\
\hline Coronary heart disease or myocardial infarction & $30,581(7.0)$ & $30,581(72.1)$ & 0 & \\
\hline Heart failure & $3,204(0.7)$ & $2,033(4.8)$ & $1,171(0.3)$ & 28.8 \\
\hline Atrial fibrillation & $15,752(3.6)$ & $15,752(37.1)$ & 0 & \\
\hline Carotid stenosis & $3,981(0.9)$ & $477(1.1)$ & $3,504(0.9)$ & 2.0 \\
\hline Diabetes mellitus & $113,613(26.0)$ & $11,695(27.6)$ & $101,918(25.9)$ & 3.8 \\
\hline Smoking, No. (\%) & $166,091(38.0)$ & $13,073(30.8)$ & $153,018(38.8)$ & 16.9 \\
\hline Missing & $9,561(2.2)$ & $780(1.8)$ & $8,781(2.2)$ & 2.9 \\
\hline \multicolumn{5}{|l|}{ Prestroke medications, No. (\%) } \\
\hline Antiplatelet & $37,178(8.5)$ & $10,217(24.1)$ & $26,961(6.8)$ & 49.3 \\
\hline Lipid-lowering therapy & $25,511(5.8)$ & $5,880(13.9)$ & $19,631(5.0)$ & 30.8 \\
\hline Antihypertensives & $179,794(41.2)$ & $21,516(50.7)$ & $158,278(40.1)$ & 21.4 \\
\hline NIHSS 16-42 & $18,919(4.3)$ & $4,611(10.9)$ & $14,308(3.6)$ & 28.4 \\
\hline Length of stay $>14 d$ & $85,126(19.5)$ & $10,449(24.6)$ & 74,677 (18.9) & 13.8 \\
\hline Missing & $6,782(1.6)$ & $755(1.8)$ & $6,027(1.5)$ & 2.4 \\
\hline Death & $1,597(0.4)$ & $544(1.3)$ & $1,053(0.3)$ & 11.2 \\
\hline Recurrence of ischemic stroke & $9,534(2.2)$ & $1,827(4.3)$ & $7,707(2.0)$ & 13.2 \\
\hline Recurrence of ischemic stroke and TIA & $11,028(2.5)$ & $1,989(4.7)$ & $9,039(2.3)$ & 13.1 \\
\hline Hemorrhagic stroke & $3,516(0.8)$ & $1,034(2.4)$ & $2,482(0.6)$ & 14.8 \\
\hline mRS 2-6 & $175,160(40.1)$ & $12,643(29.8)$ & $162,517(41.2)$ & 24.0 \\
\hline Missing & $205,636(47.1)$ & $26,680(62.9)$ & $178,956(45.4)$ & 35.7 \\
\hline
\end{tabular}

BMI, body mass index; TIA, transient ischemic attack; NIHSS, National Institute of Health Stroke Scale. 
Table 2 End points according to indication for prestroke antiplatelet use

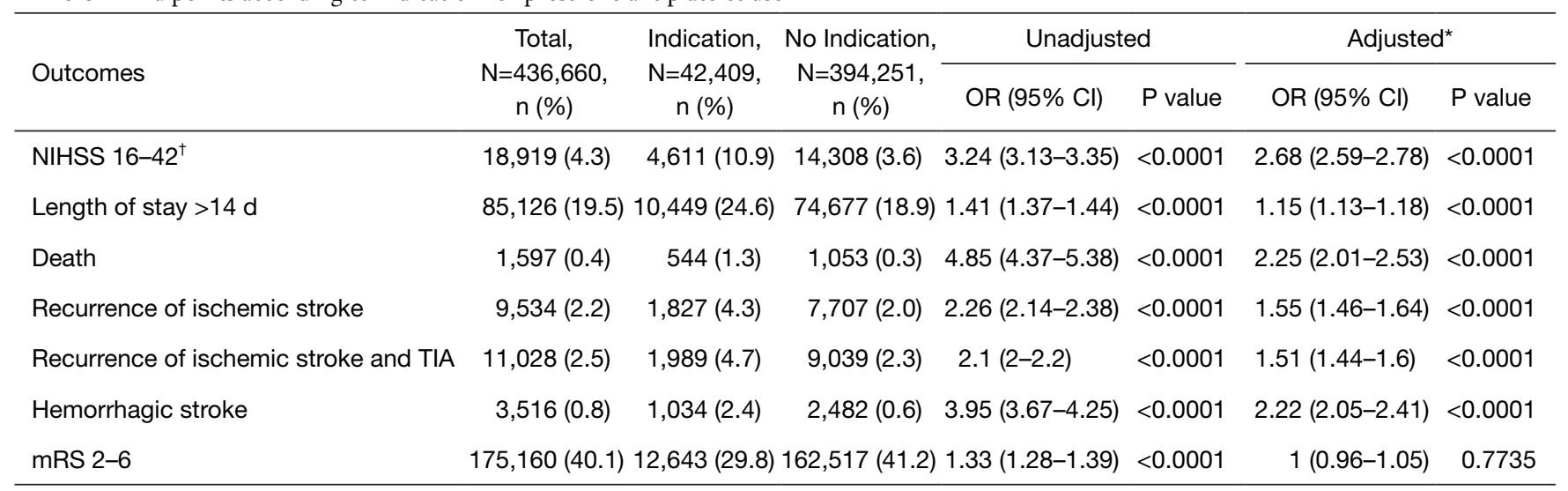

*, adjusted variables: age, sex, carotid stenosis, peripheral vascular disease, hypertension, dyslipidemia, diabetes mellitus, prestroke antiplatelet, poststroke antiplatelet, and NIHSS score; ${ }^{\dagger}$, adjusted variables: age, sex, carotid stenosis, peripheral vascular disease, hypertension, dyslipidemia, diabetes mellitus, prestroke antiplatelet, and poststroke antiplatelet. TIA, transient ischemic attack; NIHSS, National Institute of Health Stroke Scale; mRS, modified Rankin Scale.

Table 3 Outcomes by prestroke antiplatelet use and indication

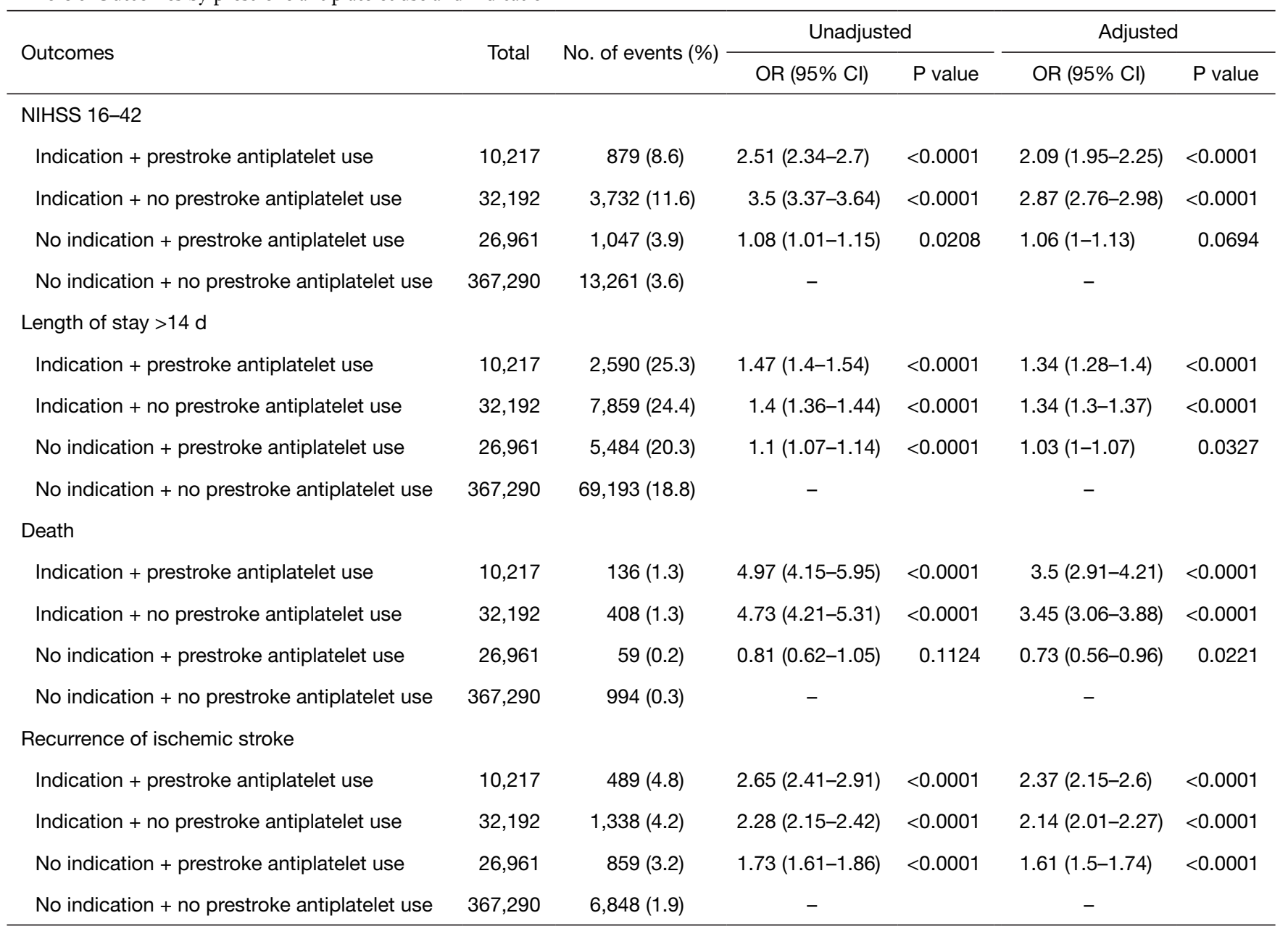

Table 3 (continued) 
Table 3 (continued)

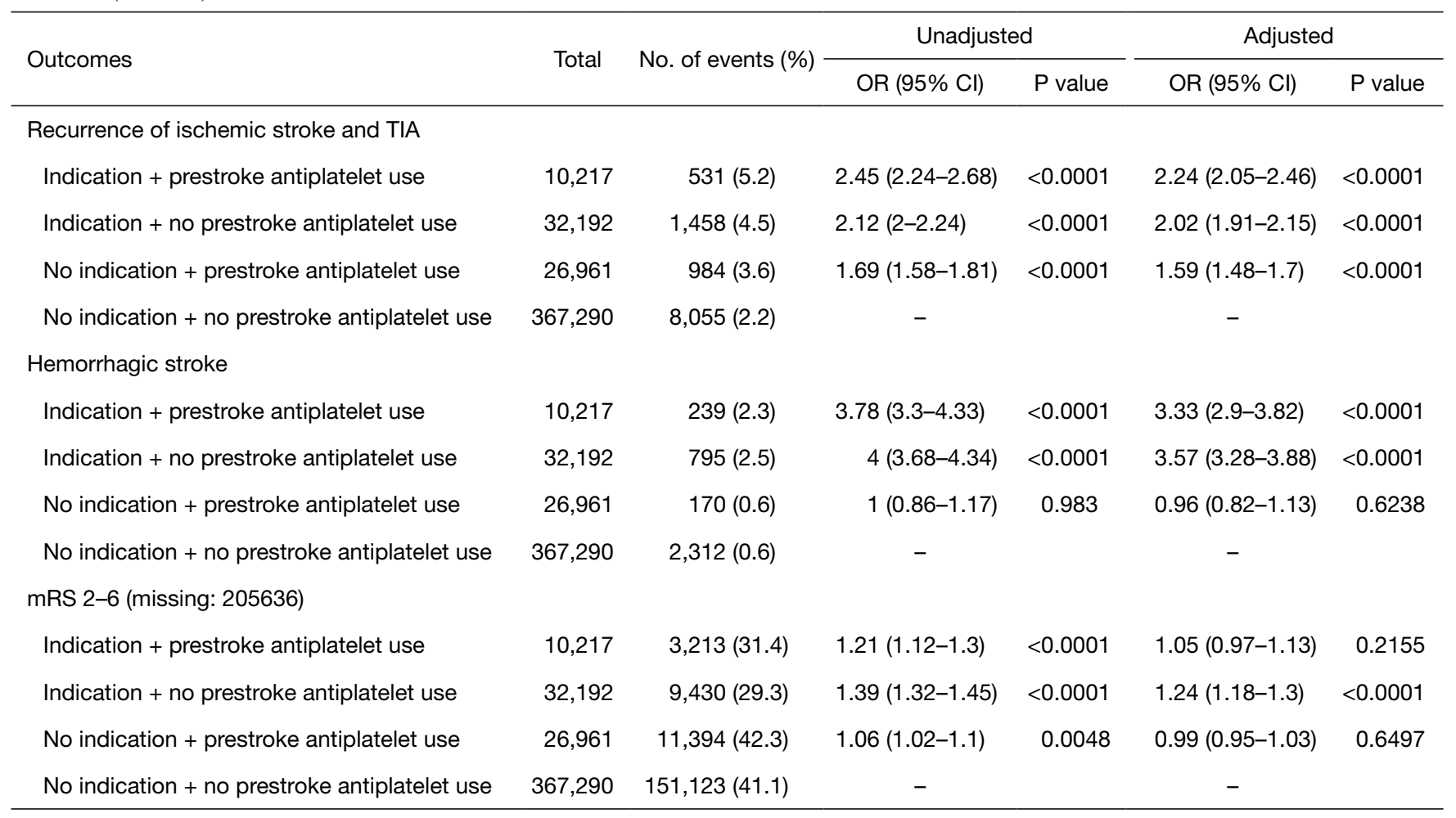

Adjusted variables: age, sex, carotid stenosis, peripheral vascular disease, hypertension, dyslipidemia, diabetes mellitus. TIA, transient ischemic attack; NIHSS, National Institute of Health Stroke Scale; mRS, modified Rankin Scale.

Second, the antiplatelet therapy was underused by those at high risk for CVD and overused by those at low risk for CVD. $75.9 \%$ of stroke patients in the indication group were not on any preventative antiplatelet medication, indicating that a proportion of them might potentially have benefited from risk stratification to reduce risks of future thrombotic events. This implied gaps in risk prediction rules or in identifying patients at high risk of cardiovascular events. Conversely, treatment with antiplatelet agents in some high vascular risk patients might have been considered inappropriate by the physician due to contra-indications such as gastrointestinal ulcers or hemorrhage or due to the patient's refusal. However, this is unlikely to account for all cases where patients did not receive the appropriate prevention. On the other hand, we found antiplatelet use before stroke in $6.8 \%$ of patients in the non-indication group. This might be due to other appropriate indications but could also reflect self-administrative treatment, which would be paid attention because of the potential bleeding hazard (12-14).

Third, our analysis revealed significant differences in rates of negative in-hospital outcomes between the two different groups of patients. Compared with patients without indication, the mortality, hospital length of stay over 14 days and NIHSS score over 16 were higher in patients with indication, which was consistent with previous studies. Besides, patients with indications were likely to suffer both recurrence of stroke or TIA and hemorrhage stroke while hospitalized. In stroke patients receiving antiplatelet therapy, ESSEN Stroke Risk Score and $\mathrm{CHA}_{2} \mathrm{DS}_{2} \mathrm{VASc}(15)$ were used to evaluate recurrence of ischemic stroke, and $\mathrm{S}_{2}$ TOP-BLEED, REACH, and $\mathrm{B}_{2} \mathrm{LEED}_{3} \mathrm{~S}$ (16) were used to predict the baseline risk of major in-hospital bleeding in clinical practice. Because of the shared risk factors in the scales of future risk ischemic and bleeding events, patients with indications for prestroke antiplatelet use were made up of groups with different balances in the risk of ischemic and bleeding events. Our results were consistent with previous reports that about $1 / 5$ patients presenting with acute coronary syndrome were at high ischemic and high bleeding risk, and they were more likely to experience poor clinical outcomes and reduced 
odds of receiving guideline-recommended therapy (17).

Fourth, in contrast to previous studies where the prevalence of antiplatelet use was high $(2,8)$, in this study, prestroke antiplatelet use only had significant protective effects on in-hospital mortality in non-indication group, and on stroke severity and in-hospital disability in the indication group. There were inconsistencies and conflicts about whether prestroke antiplatelet use could benefit patients $(2,8,18)$. Therefore, our study emphasizes the need for better identification of patients at risk, perhaps using emerging novel risk factors $(19,20)$. Chinese Stroke Association guidelines for clinical management of cerebrovascular disorders highlighted that asymptomatic carotid stenosis over $50 \%$ and asymptomatic intracranial artery stenosis were considered as high-risk factors and that antiplatelets was recommended as primary prevention (Class Iia, level of evidence C) (21).

The key advantages of our study were the much larger sample size and analysis of diverse relevant and important outcomes for patients. The previous research was limited by relatively small sample size (18) or focused on one or several outcomes alone (22-24).

Our study had several limitations. First, as a hospitalbased study, patients and hospitals might not be entirely representative of the Chinese population. Second, the definition of indications for prestroke antiplatelet use might be imperfect. We were unable to gather information about severe stenosis (over 50-70\%) of the peripheral artery, carotid artery, and intracranial artery, which were indications as well $(21,25)$. Third, to make a compromise, we regarded $\mathrm{AF}$ as an indication. Most AF patients with CHA2DS2-VASc scores of $\geq 2$ were at high risk of atherosclerosis. Antiplatelets could be used as an alternative, although there were debates on whether it would bring benefits (26-29). Besides, for a CHA2DS2-VASc score of 1 , aspirin could be considered as the primary prevention (Class IIa, level of evidence C) (30). Forth, we were not able to analyze either the class of antiplatelets, the exact duration of the therapy, and the precise dose, all of which might have effects on the prognosis of ischemic stroke. Fifth, we were unable to evaluate the prognosis in 90 days or longer, because there was no follow-up data after discharge in the CSCA database.

To conclude, for first-ever noncardiogenic ischemic stroke, patients with indications for prestroke antiplatelet therapy were more likely to have unfavorable outcomes than patients without. Prestroke antiplatelet use might be associated with lower mortality, less disability, and less severity of stroke in certain population groups. Future studies to improve risk prediction rules are needed to guide effective primary prevention of ischemic stroke to provide a more individualized approach in the future.

\section{Acknowledgments}

We thank Dr. Luanluan Sun (Cardiovascular Epidemiology Unit, Department of Public Health and Primary Care, University of Cambridge, UK) for her important intellectual contribution to the article. We thank all participating hospitals, their physicians, and nurses, and the Chinese Stroke Center Alliance Steering Committee members.

Funding: This study was funded by the National Key R\&D Program of China (No. 2018YFC1312903), National Science and Technology Major Project (No. 2017ZX09304018), Beijing Municipal Science \& Technology Commission (No. D171100003017002, Z181100001818001), and National Natural Science Foundation of China (No. 81801139).

\section{Footnote}

Reporting Checklist: The authors have completed the STROBE reporting checklist. Available at http://dx.doi. org/10.21037/atm-20-7902

Data Sharing Statement: Available at http://dx.doi. org/10.21037/atm-20-7902

Conflicts of Interest: All authors have completed the ICMJE uniform disclosure form (available at http://dx.doi. org/10.21037/atm-20-7902). The authors have no conflicts of interest to declare.

Ethical Statement: The authors are accountable for all aspects of the work in ensuring that questions related to the accuracy or integrity of any part of the work are appropriately investigated and resolved. The study was conducted in accordance with the Declaration of Helsinki (as revised in 2013). The study was approved by the central Institutional Ethics Board at Beijing Tiantan Hospital (No.: KY 2018-061-02) and individual consent for this retrospective analysis was waived.

Open Access Statement: This is an Open Access article distributed in accordance with the Creative Commons Attribution-NonCommercial-NoDerivs 4.0 International 
License (CC BY-NC-ND 4.0), which permits the noncommercial replication and distribution of the article with the strict proviso that no changes or edits are made and the original work is properly cited (including links to both the formal publication through the relevant DOI and the license). See: https://creativecommons.org/licenses/by-nc-nd/4.0/.

\section{References}

1. Wang W, Jiang B, Sun H, et al. Prevalence, Incidence, and Mortality of Stroke in China: Results from a Nationwide Population-Based Survey of 480687 Adults. Circulation 2017;135:759-71.

2. Jung JM, Choi J, Eun MY, et al. Prestroke antiplatelet agents in first-ever ischemic stroke: clinical effects. Neurology 2015;84:1080-9.

3. Dowlatshahi D, Hakim A, Fang J, Pre admission antithrombotics are associated with improved outcomes following ischaemic stroke: a cohort from the Registry of the Canadian Stroke Network. Int J Stroke 2009;4:328-334.

4. Guirguis-Blake JM, Evans CV, Senger CA, et al. Aspirin for the Primary Prevention of Cardiovascular Events: A Systematic Evidence Review for the U.S. Preventive Services Task Force. Ann Intern Med 2016;164:804-13.

5. Meschia JF, Bushnell C, Boden-Albala B, et al. Guidelines for the primary prevention of stroke: a statement for healthcare professionals from the American Heart Association/American Stroke Association. Stroke 2014;45:3754-832.

6. Arnett DK, Blumenthal RS, Albert MA, et al. 2019 ACC/AHA Guideline on the Primary Prevention of Cardiovascular Disease: A Report of the American College of Cardiology/American Heart Association Task Force on Clinical Practice Guidelines. Circulation 2019;140:e596-646.

7. Wang Y, Li Z, Wang Y, et al. Chinese Stroke Center Alliance: A national effort to improve healthcare quality for acute stroke and transient ischaemic attack: Rationale, design and preliminary findings. Stroke Vasc Neurol 2018;3:256-62.

8. Myint PK, Hellkamp AS, Fonarow GC, et al. Prior Antithrombotic Use is Associated with Favorable Mortality and Functional Outcomes in Acute Ischemic Stroke. Stroke 2016;47:2066-74.

9. Gu HQ, Li DJ, Liu C, \% ggBaseline: a SAS macro for analyzing and reporting baseline characteristics automatically in medical research. Ann Transl Med
2018;6:326.

10. Reed DM. The paradox of high risk of stroke in populations with low risk of coronary heart disease. Am J Epidemiol 1990;131:579-88.

11. Gliksman M, Wilson A. Are hemostatic factors responsible for the paradoxical risk factors for coronary heart disease and stroke? Stroke 1992;23:607-610.

12. VanWormer JJ, Greenlee RT, McBride PE, et al. Aspirin for primary prevention of CVD: Are the right people using it? J Fam Pract 2012;61:525-32.

13. Bowman L, Mafham M, Wallendszus K, et al. Effects of Aspirin for Primary Prevention in Persons with Diabetes Mellitus. N Engl J Med 2018;379:1529-39.

14. Ikeda $Y$, Shimada K, Teramoto T, et al. Low-dose aspirin for primary prevention of cardiovascular events in Japanese patients 60 years or older with atherosclerotic risk factors: a randomized clinical trial. JAMA 2014;312:2510-20.

15. Andersen SD, Gorst-Rasmussen A, Lip GYH, et al. Recurrent Stroke: The Value of the CHA2DS2VASc Score and the Essen Stroke Risk Score in a Nationwide Stroke Cohort. Stroke 2015;46:2491-7.

16. Hilkens NA, Li L, Rothwell PM, et al. External Validation of Risk Scores for Major Bleeding in a Population-Based Cohort of Transient Ischemic Attack and Ischemic Stroke Patients. Stroke 2018;49:601-6.

17. Mohamed MO, Kinnaird T, Anderson R, et al. Combinations of bleeding and ischemic risk and their association with clinical outcomes in acute coronary syndrome. Int J Cardiol 2019;290:7-14.

18. Béjot $\mathrm{Y}$, Aboa-Eboulé C, de Maistre E, et al. Prestroke antiplatelet therapy and early prognosis in stroke patients: The Dijon Stroke Registry. Eur J Neurol 2013;20:879-90.

19. Cooney MT, Dudina A, D'Agostino R, Cardiovascular risk-estimation systems in primary prevention: do they differ? Do they make a difference? Can we see the future? Circulation 2010;122:300-10.

20. Zannad F, De Backer G, Graham I, et al. Risk stratification in cardiovascular disease primary prevention - scoring systems, novel markers, and imaging techniques. Fundam Clin Pharmacol 2012;26:163-74.

21. Wang Y, Han S, Qin H, et al. Chinese Stroke Association guidelines for clinical management of cerebrovascular disorders: executive summary and 2019 update of the management of high-risk population. Stroke Vasc Neurol 2020;5:270-8.

22. Kumar N, Khera R, Pandey A, Racial Differences in Outcomes after Acute Ischemic Stroke Hospitalization in the United States. J Stroke Cerebrovasc Dis 
2016;25:1970-7.

23. Wei W, Li S, San F, et al. Retrospective analysis of prognosis and risk factors of patients with stroke by TOAST. Medicine (Baltimore) 2018;97:e0412.

24. Barlas RS, Loke YK, Mamas MA, et al. Effect of Antiplatelet Therapy (Aspirin + Dipyridamole Versus Clopidogrel) on Mortality Outcome in Ischemic Stroke. Am J Cardiol 2018;122:1085-90.

25. Aboyans V, Ricco JB, Bartelink M-LEL, et al. 2017 ESC Guidelines on the Diagnosis and Treatment of Peripheral Arterial Diseases, in collaboration with the European Society for Vascular Surgery (ESVS): Document covering atherosclerotic disease of extracranial carotid and vertebral, mesenteric, renal, upper and lower extremity arteries Endorsed by: the European Stroke Organization (ESO) The Task Force for the Diagnosis and Treatment of Peripheral Arterial Diseases of the European Society of Cardiology (ESC) and of the European Society for Vascular Surgery (ESVS). Eur Heart J 2018;39:763-816.

Cite this article as: $\mathrm{Xu} Y Y, \mathrm{Gu} H Q, \mathrm{Li} Z \mathrm{Z}$, Xiong YY, Zhou Q, Liu LP, Zhao XQ, Wang YL, Meng X, Wang YJ; on behalf of the CSCA Investigators. In-hospital prognosis of first-ever noncardiogenic ischemic stroke in patients with and without indication for prestroke antiplatelet therapy: Chinese Stroke Center Alliance. Ann Transl Med 2021;9(8):626. doi: 10.21037/atm-20-7902
26. Aguilar M, Hart R. Antiplatelet therapy for preventing stroke in patients with non-valvular atrial fibrillation and no previous history of stroke or transient ischemic attacks. Cochrane database Syst Rev 2005;(4):CD001925.

27. Stroke Prevention in Atrial Fibrillation Study. Final results. Circulation 1991;84:527-39.

28. Connolly SJ, Pogue J, Hart RG, et al. Effect of clopidogrel added to aspirin in patients with atrial fibrillation. $N$ Engl J Med 2009;360:2066-78.

29. Connolly S, Pogue J, Hart R, et al. Clopidogrel plus aspirin versus oral anticoagulation for atrial fibrillation in the Atrial fibrillation Clopidogrel Trial with Irbesartan for prevention of Vascular Events (ACTIVE W): a randomised controlled trial. Lancet 2006;367:1903-12.

30. January CT, Wann LS, Alpert JS, et al. 2014 AHA/ ACC/HRS guideline for the management of patients with atrial fibrillation: a report of the American College of Cardiology/American Heart Association Task Force on practice guidelines and the Heart Rhythm Society. Circulation 2014;130:e199-267. 